\title{
De HÁBITAT II a HÁBITAT III. Construyendo con recursos escasos en Latinoamérica
}

\author{
From HABITAT II to HABITAT III. Building with scarce resources in \\ Latin America
}

\author{
Rafael Llácer Pantión* \\ Fecha de recepción: 10-07-2017 - Fecha de aceptación: 22-08-2017 \\ Hábitat y Sociedad (ISSN 2173-125X), n. ${ }^{\circ} 10$, noviembre de 2017, pp. 335-340. \\ http://dx.doi.org/10.12795/HabitatySociedad.2017.i10.19
}

\begin{abstract}
Publication that includes the set of experiences and works that Dr. Julián Salas has been developing for decades in very low cost housing in Latin America. The topics are organized into six monographic chapters, beginning with an orderly presentation of what happened since the World Conference on Human Settlements (HABITAT II) of 1996 to nowadays and the concept of Basic Habitability as a tool to work against poverty In terms of habitat. The influence of the land is focused through the reality of the urban landings, exposing the analysis of the data of a survey carried out in 18 Latin American countries. The concern for the improvement of slums is reflected in the presentation of the interventions of Ciudad Sandino (Nicaragua) and Las Palmas in San Salvador. The presence of the materials is present in the influence in the analysis of the costs of the house and in the advantages of which denominates like other materials in the Solutions for Very Low Cost Dwelling. Proposals for technologies and industrialization with limited resources close this latest publication of Professor Salas Serrano.
\end{abstract}

\section{Key words}

Basic habitability; Very Low Cost Dwelling; Informal settlements; Limited resources; Latin America

\begin{abstract}
Resumen
Publicación que recoge el conjunto de experiencias y trabajos que el Dr. Julián Salas viene desarrollando desde hace décadas en temas de viviendas de muy bajo coste en Latinoamérica. Los temas se organizan en forma de seis capítulos monográficos, iniciando el recorrido una ordenada exposición de lo acontecido desde la Conferencia Mundial sobre los Asentamientos Humanos (HÁBITAT II) de 1996 hasta nuestros días y el concepto de habitabilidad básica como herramienta de lucha contra la pobreza en materia de hábitat. La influencia del suelo se enfoca a través de la realidad de las tomas de tierras urbanas, exponiendo el análisis de los datos de una encuesta realizada en 18 países latinoamericanos. La preocupación por la mejora de tugurios queda recogida por la exposición de las intervenciones de Ciudad Sandino (Nicaragua) y de Las Palmas en San Salvador. La presencia de los materiales está presente en la influencia en los análisis de los costes de la vivienda y en las ventajas de los que denomina como otros materiales en las soluciones para viviendas de muy bajo coste. Propuestas de tecnologías e industrialización con recursos escasos cierran esta última publicación del profesor Salas Serrano.
\end{abstract}

\section{Pallabras clave}

Habitabilidad básica; Vivienda de muy bajo coste; Asentamientos informales; Recursos escasos; Latinoamérica

\footnotetext{
* Profesor del Departamento Construcciones Arquitectónicas II. Escuela Técnica Superior de Ingeniería de Edificación, Universidad de Sevilla. Avda. Reina Mercedes, 4A, 41012 Sevilla. C.e.: rllacer@us.es.
} 
Salas Serrano, J. (2016) De HABITAT II a HABITAT III. Construyendo con recursos escasos en Latinoamérica. Madrid: Ministerio de Fomento. Instituto Juan de Herrera, ETSAM, UPM

Obra publicada por la Dirección General de Arquitectura, Vivienda y Suelo, del Ministerio de Fomento, que plasma el trabajo que el autor viene desarrollando desde hace décadas en temas de habitabilidad y asequibilidad de la vivienda. Julián Salas ha dedicado gran parte de su vida profesional a temas relacionados con la construcción de viviendas de muy bajo coste. Creó y coordinó el Sub-programa Iberoamericano CYTED sobre "Tecnologías y materiales para viviendas de muy bajo coste en Latinoamérica" y entre sus publicaciones cabe destacar Contra el Hambre de Vivienda: soluciones tecnológicas latinoamericanas (1993) y La Industrialización Posible de la Vivienda en Latinoamérica (2000). Igualmente, ha realizado más de doscientos artículos y trabajos científicos. Ahora, con esta última publicación aporta una visión integradora de las mejoras existentes enfocadas a los problemas habitacionales y un conjunto de técnicas concretas para aplicar a los mismos.

Estructurado en seis capítulos, el libro se abre con una ordenada exposición de lo acontecido desde la Conferencia Mundial sobre los Asentamientos Humanos (HÁBITAT II) de 1996 hasta nuestros días. Entre otros, se encuentran los contenidos concretos del concepto habitabilidad básica, la formalización de cuadros sinópticos con los objetivos de desarrollo sostenible que afectan al hábitat y al medioambiente, datos estadísticos sobre el problema del acceso al agua potable y al saneamiento y esquemas de las tendencias del futuro inmediato.

En este primer apartado, el autor va exponiendo los diferentes tipos de asentamientos que se producen en medios rurales y urbanos, los campamentos de emergencia, los tugurios o slums (más de $2500 \mathrm{mi}$ llones de personas habitan en ellos) y datos cuantitativos sobre formas de vida en condiciones extremas, así como la realidad del "hambre de vivienda" en Latinoamérica.

Estas líneas reflejan la paradoja entre el derecho a la vivienda en los textos legales y el negocio que genera al convertirse en un bien no asequible. En este sentido, incluye una tabla que recoge los textos articulados en relación con la vivienda en Latinoamérica.

En el segundo capítulo, "La importancia del suelo en la solución del hábitat humano", el autor expone la realidad de las tomas de tierras urbanas. Para ello, lleva a cabo una encuesta a 70 especialistas de 18 países latinoamericanos, cuyos resultados y conclusiones han constituido la publicación Las tomas de tierras urbanas en Latinoamérica hoy: ¿Problema o Solución? (Salas et al., 2010). A lo largo de varios apartados se ofrece una extensa información sobre los diferentes organismos latinoamericanos responsables de los temas de vivienda, programas de ayuda en relación con los asentamientos irregulares, leyes vigentes, así como una detallada y amplia información basada en las respuestas a las mencionadas encuestas. El autor enumera una serie de conclusiones, recordando que de las cuatro etapas que propugna el Instituto de Cooperación en Habitabilidad Básica (ICHaB), como son la elección del suelo apropiado, la parcelación racional, la urbanización y la edificación, las tomas ilegales encuentran especiales dificultades en la primera y aquellos con menos recursos centran sus mayores esfuerzos en la edificación. Las dos primeras etapas, que son el verdadero germen 
para cubrir las necesidades de habitabilidad y que apenas tienen costes económicos, deben requerir intervención pública y de técnicos cualificados.

Se completa el capítulo con dos amplios apartados en los que se expone el análisis de dos casos reales de gran repercusión: la toma de Peñalolén en Santiago de Chile y la Ocupación Guiada en el Alto Trujillo en Perú.

En el tercer capítulo, titulado "Mejora de tugurios: prioridad latinoamericana en materia de habitabilidad básica $(\mathrm{HaB})$ " destacan dos extensos subcapítulos en los que se desarrollan dos importantes intervenciones como son la de Ciudad Sandino (Nicaragua) y la de Las Palmas en San Salvador.

La primera intervención, denominada como la mejora de "caso a caso y casa a casa" de 400 viviendas precarias, ilustra una experiencia que puede servir como modelo, una experiencia que demuestra que "las políticas simplificadoras del tipo solución única para todos" pueden ser oportunidades fallidas en la lucha contra la pobreza. Mediante una metodología de "caso a caso y casa a casa" dividida en etapas de dos años, se realizó una importante mejora a pesar de las dificultades que conllevaba el no actuar desde la rutina.

Por su parte, la segunda intervención sirve como ejemplo de las experiencias llevadas a cabo por la Fundación Salvadoreña de Desarrollo y Vivienda Mínima (FUNDASAL). Esta institución privada, que durante los 30 años de existencia lleva construidas más de 30000 viviendas en El Salvador, lleva su actividad más allá, implicándose en la asistencia social y la organización de la construcción.

El cuarto capítulo recoge en uno de sus apartados, titulado "Grandes retos para la industria de materiales básicos de construcción”, una descomposición de costes de construcción basada en realizaciones de viviendas sociales en Colombia, Brasil, Chile y El Salvador. El estudio económico (diferenciando terreno, urbanización, construcción y costes indirectos) señala cómo las variaciones de los costes de construcción por metro cuadrado oscilan entre 102 y 130,5 dólares (USA).

El autor plantea "con carácter netamente pedagógico" un posicionamiento conceptual planteando lo que denomina la "Regla de las tres $(A+B+C)$ ": Financiación, Terreno y Construcción, que, a su vez, se dividen en otros tres aspectos. El conjunto de las tres subdivisiones de cada uno de los tres grandes conceptos permite extraer algunas conclusiones según el proyecto que se realice: en situación de pobreza extrema o emergencia, en países con IDH medio/bajo o en países de desarrollo alto.

También aporta datos estadísticos sobre el consumo del cemento en relación con el sector "informal", ámbito donde se produce el consumo mayoritario de materiales básicos de edificación o la participación desmedida de las importaciones. Todo ello repercute negativamente en la propia estructura de costes.

Ante este diagnóstico propone algunos planteamientos como es la idea de que los gobiernos deben fomentar y apoyar "el suministro de materiales básicos de construcción de producción local, asequibles y duraderos".

A partir de este punto el autor recorre las diferentes técnicas, utilizadas en experiencias contrastadas, de utilización de materiales con grandes posibilidades en la construcción de viviendas de muy bajo costo (VMBC), como son la tierra, el hormigón, el ferrocemento, la madera, así como la utilización de desechos agroindustriales. 
El libro concluye con dos capítulos sobre el uso de las tecnologías. En ellos, el autor pone el foco en la utilidad de las mismas para encontrar soluciones a la vivienda de muy bajo coste y reflexiona sobre los conceptos de tecnología, transferencia y difusión en el sector del hábitat popular.

Sobre la tecnología, propone acotar seis aspectos de la misma: la tecnología como producto, su mercado, su distribución, promoción, precio y negociación de su transferencia. También hace referencia a lo que califica como "saltos tecnológicos" e incluye experiencias en Uruguay, Argentina, Brasil, Nicaragua... Todas ellas representan ingeniosas aplicaciones del ladrillo cerámico.

El acceso a la ciudad, según lo define Pedro Abramo, se realiza de tres formas: la que sigue la lógica del mercado, la que se guía por la formalidad del sector público y la que obedece a una lógica impuesta por la necesidad, el sector informal. En cada uno de estos tres ámbitos es posible detectar una serie de procedimientos distintos de gestión y construcción del hábitat. En los procesos de construcción y en los elementos constructivos enfocados al hábitat de los asentamientos informales, los "componentes neutros" son aquellos elementos tecnológicos proyectados de manera que puedan resultar autónomos o agregables en diversas situaciones. Por ello, Salas expone las posibilidades que ofrecen el diseño, construcción y producción del hábitat mediante el empleo intensivo de estos componentes. En este sentido, la ventana de hormigón desarrollada por el Centro Experimental de la Vivienda Económica (CEVE), de Córdoba, Argentina, representa uno de los ejemplos más claros de lo que es un componente neutro.

$\mathrm{El}$ autor destaca que

el continuo aumento de la población latinoamericana, con tasa de crecimiento del 1,3\% anual [...], resulta muy superior al resto de los continentes. Se prevé que alcance los 670 millones de habitantes en 2018. Este fenómeno requiere y propicia mayores ritmos de construcción, que sean a la vez capaces de reactivar la abundante mano de obra cesante o semi-empleada, un grupo de población que, además, está en gran parte necesitado de soluciones habitacionales.

Por estos motivos, defiende el uso de tecnologías sencillas que se adapten a diferentes entornos y ofrezcan mejores prestaciones que los métodos tradicionales. Componentes neutros y tecnologías compatibles se presentan, así, como posibles vías para abordar el complejo problema de los asentamientos informales.

La convivencia de lo informal y lo planificado, de la industrialización y la autoconstrucción, son ilustradas con la experiencia del Proyecto Experimental de Vivienda (PREVI) en un apartado propio. Este proyecto, realizado en Lima y comenzado en 1967, proporciona datos suficientes para considerarlo un banco de pruebas donde extraer algunas conclusiones, ya que todas las propuestas presentadas al concurso han sido construidas y, además, en las bases de la convocatoria se hacía referencia al empleo de procesos industrializados.

Estas bases contemplaron también el crecimiento en el tiempo (por ello construyeron viviendas susceptibles de crecer de manera adecuada), la racionalización constructiva y la prefabricación de elementos ligeros. Por otro lado, los equipos que participaron lo hicieron llegados de diferentes países y presentaron elementos prefabricados de variada envergadura. Pasado el tiempo, resulta evidente que la capacidad 
de adaptación de la industrialización ligera ha sido positiva, pero también tras comprobar las modificaciones llevadas a cabo en los proyectos mediante técnicas tradicionales surge una pregunta: por qué estas tecnologías no han prendido en el tejido industrial local. El crecimiento económico de Perú hace previsible el aumento de la demanda de viviendas y en esta nueva situación, según Salas, podría ser factible el tener en cuenta las experiencias de PREVI, tanto para este país como para otras zonas de Latinoamérica.

Por último, en el sexto capítulo, el autor comienza con una reflexión propia: el empleo de "lo mejor y lo más barato" ha sido el principal criterio que ha seguido en su dilatada trayectoria. A lo largo del mismo, se presentan una selección de soluciones, sistemas constructivos o actuaciones que el autor ha experimentado, empezando por un elemento tan básico y simbólico como es el techo y las conclusiones extraídas de la publicación Un techo para vivir, labor colectiva de decenas de autores coordinada por Pedro de Lorenzo (2005).

Así, se aborda la producción de bloques de hormigón prefabricado, el sistema "Tabibloc" o el sistema de elementos prefabricados Sandino, uno de los más utilizados de Latinoamérica para viviendas de una altura e incluso dos, con pequeñas adaptaciones. También se expone la experiencia del denominado "Gran Galpón”, eficaz solución de Carlos González Lobo, que materializa su idea de que la vivienda de interés social debe medirse y compararse en función del número de metros cúbicos construidos. Concluye esta serie el sistema BENUMA (Ferrero \& Gargatni, 2013); en este caso, Salas describe con claridad, combinando texto y fotografía, un proceso constructivo que ha servido para llevar a cabo la construcción de numerosas viviendas de Argentina.

El autor finaliza reservando un espacio para la reflexión sobre la enseñanza de la habitabilidad básica $(\mathrm{HaB})$ y cómo la metodología utilizada para la docencia de la arquitectura dominante - la que se enseña en escuelas y facultades - se conforma a partir de lo observado en países desarrollados. En este sentido, Naciones Unidas para el Hábitat nos recuerda que "hoy, por cada habitante del mundo desarrollado que vive en ciudades, dos lo hacen en ciudades del Tercer Mundo, y para el 2030 esta proporción se duplicará, será de cuatro a uno".

A modo de epílogo, el autor expone una síntesis de su pensamiento. Arquitectura, desarrollo humano, enseñanza y habitabilidad básica son conceptos que fluyen a lo largo de estas últimas páginas y que han sido referencia en su experiencia, a lo largo de los más de veinte años de enseñanza de posgrado en "Cooperación para el Desarrollo en Habitabilidad Básica en Asentamientos Precarios”.

El subsecretario de Ministerio de Fomento, Mario Garcés Sanagustín, califica el libro en su prólogo como "elemento de reflexión, análisis y, en su caso, actuación”. En definitiva, se trata de una obra que incluye diferentes enfoques y situaciones para construir con escasos recursos, con reflexiones y análisis de múltiples soluciones y experiencias, así como un compendio y una ordenación del conocimiento adquirido a lo largo de una larga y fructífera trayectoria profesional. 


\section{Referencias}

Salas, J., et al. (2010). Las Tomas de tierras urbanas en Latinoamérica hoy: ¿Problema o Solución? Madrid: Ministerio de Vivienda.

Lorenzo Gálligo, P. (coord.) (2005). Un techo para vivir. Tecnologías para viviendas de producción social en Amé- rica Latina. Barcelona: Ediciones UPC, CYTED, subprograma XIV, proyecto XIV.3 y XIV.5.

Pipa, D. y Ferrero, A. (2013). Manual del sistema UMA. Villa Mercedes: Editorial Tabaquillo, Villa Mercedes.

Llácer Pantión, R. (2017). De HÁBITAT II a HÁBITAT III. Construyendo con recursos escasos en Latinoamérica. Hábitat y Sociedad, 10, 335-340.

<http://dx.doi.org/10.12795/HabitatySociedad.2017.i10.19> 